Reprinted from:

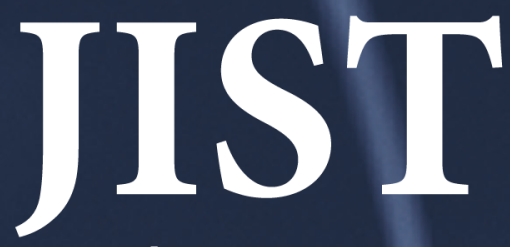

Vol. 52, No. 3 May/June 2008

Journal of

Imaging Science

and Technology

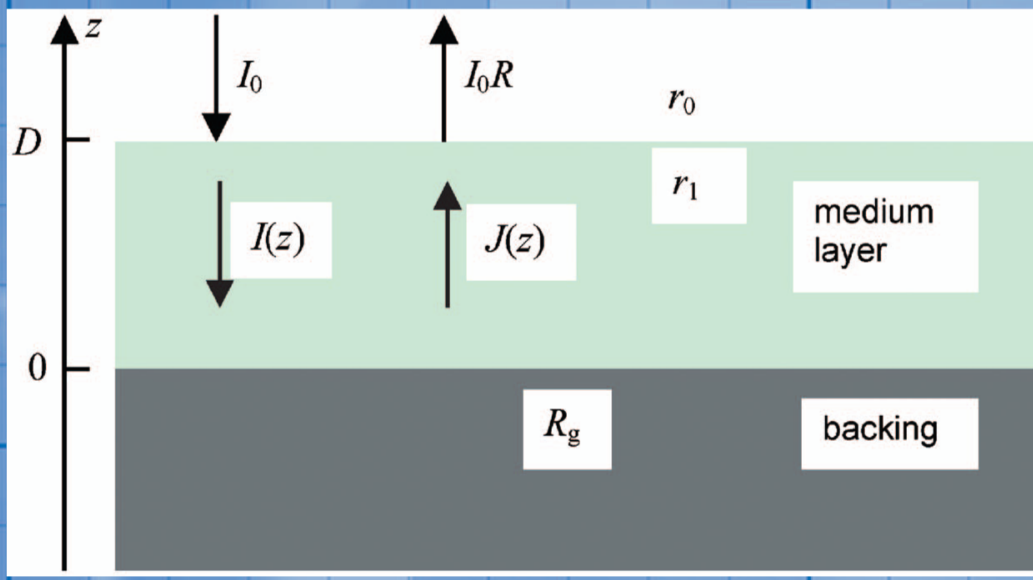

Society for Imaging Science and Technology 



\title{
Study on the Charge Control Mechanism of Charge Control Agents
}

\author{
J. Mizuguchi, A. Hitachi, Y. Sato and K. Uta \\ Graduate School of Engineering, Yokohama National University, 240-8501 Yokohama, Japan \\ E-mail:mizu-j@ynu.ac.jp
}

\begin{abstract}
A charge control mechanism of charge control agents (CCAs) has been proposed in the present investigation that assumes an appreciable temperature increase at the "toner/carrier" interface due to triboelectrification. A further assumption is that the CCA is present on the surface of both toner and carrier. Because of local heating, the electrical conductivity of CCAs is increased remarkably to give a conductive channel, through which the carrier flow occurs effectively to charge the toner. These two assumptions have experimentally been verified. Especially, local heating of up to $\sim 100^{\circ} \mathrm{C}$ has been confirmed by using a pigment marker that changes its color from black to red. Around this temperature, the electrical conductivity of CCAs is also found to increase significantly by one to three orders of magnitude as compared with that at room temperature. () 2008 Society for Imaging Science and Technology. [DOI: 10.2352/J.ImagingSci.Technol.(2008)52:3(030506)]
\end{abstract}

\section{INTRODUCTION}

Charge control agents (CCAs) are widely used in electrophotography to create a desired charge level and polarity. A number of investigations have been carried out on toner charging and the charge control mechanism, on the basis of the effective electric field, ${ }^{1,2}$ the work function, ${ }^{3}$ mass transfer, ${ }^{4}$ and charge transfer. ${ }^{5}$ However, no clear-cut explanation has yet been established.

In the present paper, we propose a novel model that assumes an appreciable temperature increase at the "toner/ carrier" interface due to triboelectrification. ${ }^{6,7}$ Because of the present local heating, the electrical conductivity of the CCA (which resides on the surface of both toner and carrier) is increased remarkably to give a conductive channel, through which the carrier flow occurs effectively to charge the toner.

We assume in the first place that the toner charge arises from the difference in work function between toner and carrier, and that the CCA resides on the surface of both toner and carrier, although the CCA is almost always used as an internal agent inside the toner. However, the existence of the CCA on the surface of toners and carriers in practical machines has been verified experimentally by Nash et al. ${ }^{3}$ and by Oguchi et al. ${ }^{4}$ We believe that the triboelectric potential caused by the difference in work function between toner and carrier, and is similar to the contact potential of a thermocouple generated at the interface between two different met-

Received Sep. 10, 2007; accepted for publication Feb. 18, 2008; published online May 19, 2008.

$1062-3701 / 2008 / 52(3) / 030506 / 5 / \$ 20.00$ als (A and B), i.e., $\Delta \phi=\phi_{A}-\phi_{B}$, where $\phi$ denotes the work function. That is, electron transfer occurs spontaneously from metal A with a higher work function to metal B with a lower work function. As a result, the contact potential appears at the contact when the electrochemical potential (i.e., Fermi level) becomes equal on both sides of the interface. However, simple contact of two different metals does not work as a thermocouple because of oxides at the metal surfaces, which disturb the electron flow, or because of a rough surface that reduces the effective contact area. Therefore, flame or electric welding is often used to contact two metals using a flux. The flux is assumed to serve as a material to remove oxide layers as well as to increase the wetting properties. We propose that the CCA plays a similar role to that of flux and bridges the toner and the carrier to facilitate the charge flow.

Figure 1 is a schematic illustration of the model, showing the toner and the carrier bridged by CCA particles. Our model assumes that the temperature at the toner/carrier interface increases significantly upon collision in the triboelectrification process. That is, the kinetic energy of both toner and carrier can be converted into thermal energy as soon as the impact takes place. Next, the CCA instantly heats up to a certain temperature, say $100^{\circ} \mathrm{C}$. Since the CCA is an organic semiconductor, its electrical conductivity increases exponentially to form conductive channels at the interface between the toner and the carrier. This greatly facilitates the charge flow, leading to charge saturation of the toner. The charge saturation is primarily determined by the work function of the polymers that cover the surface of

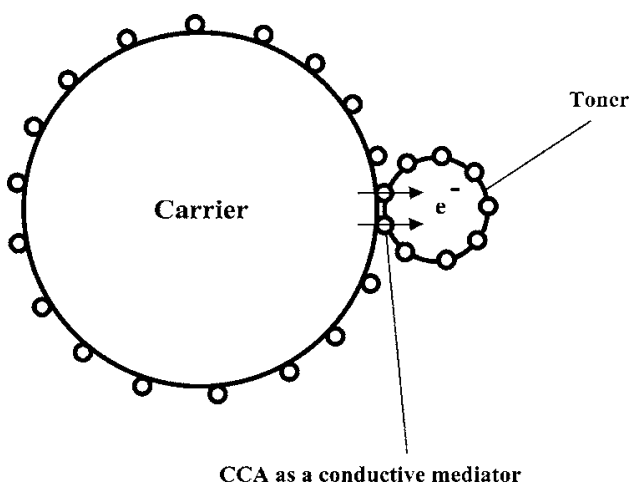

Figure 1. Charge transfer at the toner/carrier interface upon triboelectrification. 
<smiles>CCCCCCC[N+](CCCCCC)(c1ccccc1)C(CCCC)(CCCCC)CCCCC</smiles>

(a) P-51

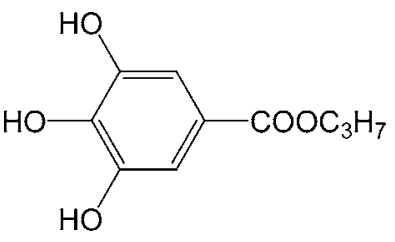

(b) PG

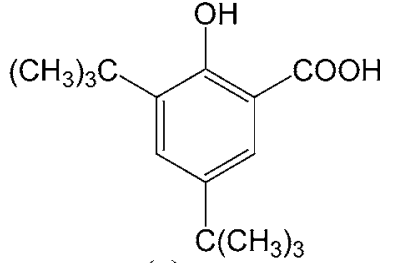

(c) TBS
Figure 2. Molecular conformation of CCAs: (a) tributylbenzylammonium naphthol-4-sulfonate (P-51), (b) n-propyl gallate (PG), and (c) 3,5-tert butylsalicylate (TBS).

toner and carrier, and secondarily by the coverage of the CCA. Complete coverage of the toner or carrier surface with CCA leads to no charging because no effective difference in work function exists between the toner and the carrier, i.e., $\Delta \phi \approx 0$, where $\phi$ again denotes the work function.

In order to prove our proposed model, the following points must be proven: (a) local heating at the toner/carrier interface upon collision; (b) temperature dependence of the electrical conductivity of CCA; and (c) determination of CCA concentration on the carrier.

\section{EXPERIMENT}

\section{CCAs}

Figure 2 shows the CCAs used for the present experiments. Those CCAs were selected which possess well-defined melting points, because the "melt/recrystallized" samples lead to more reproducible results than those in the form of tablets (i.e., pressed powders). Tributylbenzylammoniumnaphthol4-sulfonate (P-51 from Orient Chemical Industries) is a typical CCA of the positive type, whereas $n$-propyl gallate (PG) and 3,5-tert-butylsalicylate (TBS) are of the negative type, both of which were purchased from API Corporation. The crystal structures of these compounds have been published in the literature: $\mathrm{P}-51,{ }^{8,9} \mathrm{PG},{ }^{10-12}$ and TBS. ${ }^{13}$

\section{Electrical Measurements of the Temperature Dependence of CCAs}

The electrical measurements of CCAs in the form of tablets often give scattered results. Therefore, we employed the following method. We first melt CCA in a glass capillary $(2.3 \mathrm{~mm}$ in diameter: Figure 3), which includes $\mathrm{Cu}$ electrodes, and then crystallize the melt by gradual cooling. Great care was taken to keep the sample entirely transparent in the cooling process in order to avoid crack formation in the crystals, which results partly in a powder-like material. Electrical measurements were then made in air over the temperature range between room temperature and $150^{\circ} \mathrm{C}$, with a heating rate of $3^{\circ} \mathrm{C} / \mathrm{min}$.

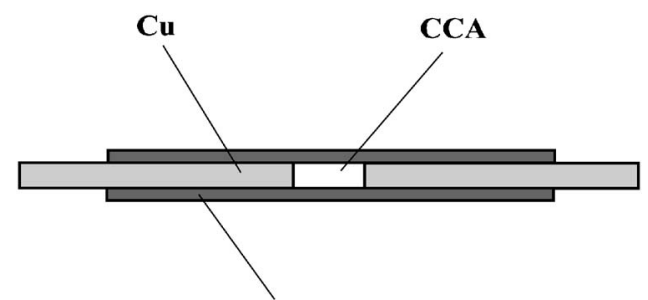

Glass Capillary

Figure 3. Experimental setup for measurements of the electric conductivity as a function of temperature.
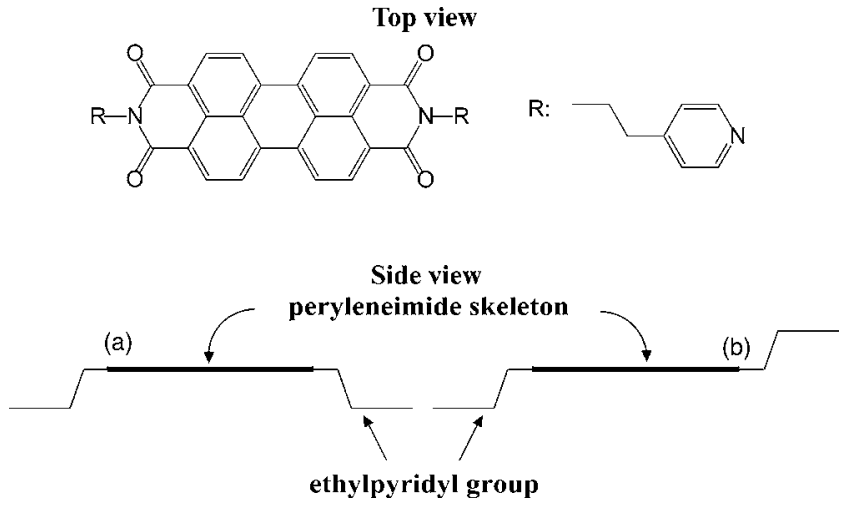

Figure 4. Molecular structure of 4-pyridylethylperyleneimide (EPP): (a) cis form and (b) trans form.

\section{Temperature Marker for the Determination of the Local Temperature}

Figure 4 shows the molecular structure of an ethylpyridylperyleneimide derivative (EPP) used as the temperature marker. EPP is known to have two structural isomers in the solid state: a cis form (red) and a trans form (black). ${ }^{14}$ In the cis form, ${ }^{15}$ ethylpyridyl rings are on the same side with respect to the peryleneimide skeleton, whereas they are positioned across the skeleton from each other in the trans form. The trans form ${ }^{16,17}$ can be transformed into the cis form by heating above $100^{\circ} \mathrm{C}$, to induce the molecular rearrangement. This phase transition induces a remarkable color change from black to vivid red. The black color is composed of two absorption bands: one is due to individual molecules and the other is caused by intermolecular interactions between two transition dipoles. The latter component is closely related to the molecular arrangement. Details on the two different colors are given in Ref. 14. Accordingly, EPP can serve as a sensitive marker to examine the local temperature at the toner/carrier interface upon collision.

EPP was synthesized by heating perylene-3,4:9,10tetracarbocarboxylic dianhydride and 4-(2-aminoethyl)pyridine at $130^{\circ} \mathrm{C}$ in water for $5 \mathrm{~h}$. The dark and viscous cake was isolated and then dried under vacuum at room temperature for several days.

Pseudo toners $(8.5 \pm 0.5 \mu \mathrm{m}$ in diameter $)$ made of styrene-acrylate polymer and Teflon ${ }^{\oplus}$-coated carriers (70 $\mu \mathrm{m}$ in diameter) were used in the present experiments; $0.5 \mathrm{~g}$ of pseudo toner $(8.5 \pm 0.5 \mu \mathrm{m}$ in diameter), $9.5 \mathrm{~g}$ of the Teflon ${ }^{\circledast}$-coated carrier (70 $\mu \mathrm{m}$ in diameter), and $10 \mathrm{mg}$ 


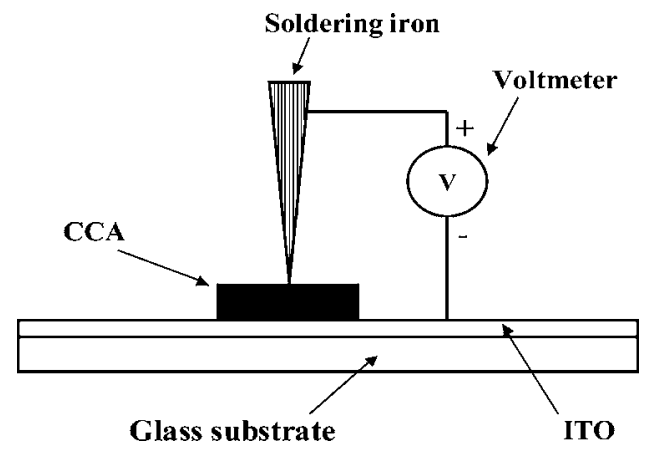

Figure 5. Experimental setup for measurements of the Seebeck coefficient.

of EPP were charged in a polyethylene bottle of $100 \mathrm{ml}$. The bottle was then rotated at $100 \mathrm{rpm}$ for 30 and $60 \mathrm{~min}$. After that, the diffuse reflectance spectra of the mixtures of toners and carriers were measured.

\section{Samples for Measurements of the Work Function}

Existence of CCA on both the toner and carrier surfaces during electrification has been examined by measuring the work function of the fresh carrier and the carrier triboelectrified with pseudo toners, which include $0.5 \%$ of CCA (triphenyl-pararosaniline sulfate). If a difference in work function is found, this can be a sign for the transfer of CCA embedded in the toner into the carrier surface.

\section{Experimental Setup for Measurements of the Seebeck Coefficient}

Figure 5 shows the experimental setup for measurements of the Seebeck coefficient for the determination of the charge carrier. ${ }^{18}$ This method measures thermoelectric power that appears between hot and cold ends of a material. The hot end is made by a soldering iron with a small spring on top maintained at $100^{\circ} \mathrm{C}$, whereas the cold end is an (indium tin oxide) electrode at room temperature. If the potential of the hot end is positive, then the carriers are electrons. Similarly, if the potential is negative, the charge carriers are holes. The CCA samples were prepared by melting/recrystallizing powdered CCA on an ITO-coated glass substrate (thickness: about $100 \mu \mathrm{m})$.

\section{Equipment}

Diffuse reflectance spectra of the mixture of toners and carriers were measured on a UV-2400PC spectrophotometer together with an integrating sphere attachment (ISR-240A from Shimadzu). The electrical conductivity was measured on CCAs by means of a Keithley 6514 system electrometer. The work function of carriers was measured by a "photoelectron spectrometer in air” from Riken Keiki Co., Ltd.

\section{RESULTS AND DISCUSSION}

\section{Local Temperature at the Toner/Carrier Interface}

Figure 6 shows the diffuse reflectance spectra of the tonercarrier mixture as a function of rolling time. As evident from the spectra, the longest wavelength band in the black spectrum begins to disappear due to triboelectrification in $30 \mathrm{~min}$ and more significantly in $60 \mathrm{~min}$, showing a color

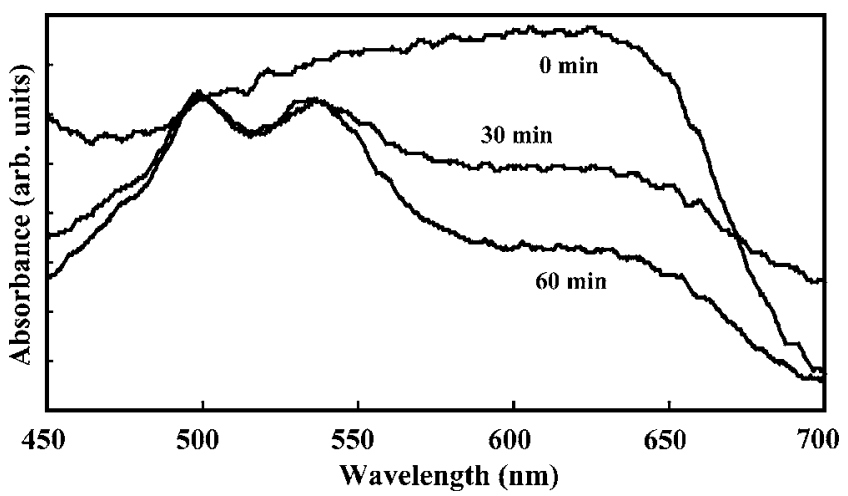

Figure 6. Diffuse reflectance spectra of the toner-carrier mixture before and after rolling.

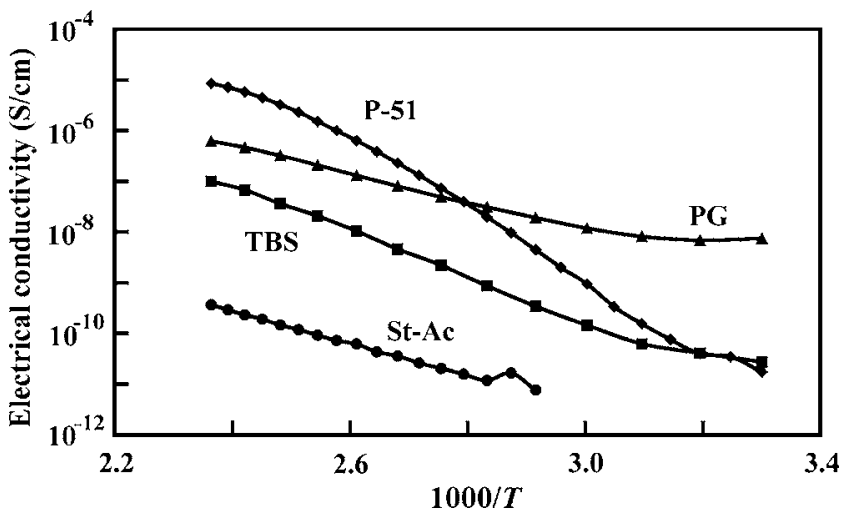

Figure 7. Arrhenius plot (log $\sigma$ vs $1 / T$ ) for various CCAs and styreneacryl polymer where $\sigma$ and $T$ denote electrical conductivity and temperature, respectively.

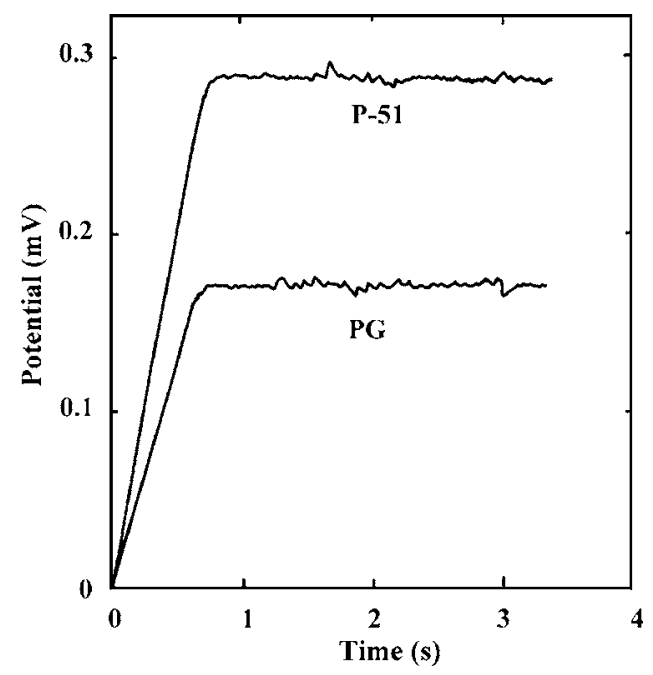

Figure 8. Seebeck potential as a function of time for P-51 and PG.

change from black to red. This indicates that the kinetic energy of both toner and carrier is transformed into thermal energy on impact. As a result, the local temperature at the toner/carrier interface exceeded $100^{\circ} \mathrm{C}$, causing the phase change to occur that induces the "trans/cis" molecular rearrangement. It is likely then, that the CCA that exists between 
the toner and the carrier (see Figure 1) is heated above $100^{\circ} \mathrm{C}$, leading to the formation of conductive channels between toner and carrier, as shown below.

Evidence of the temperature increase of toners in a single-component system is also given in the Appendix showing SEM (scanning electron microscope) pictures before and after several ten thousands' printing.

\section{Temperature Dependence of Electrical Conductivity of $C C A$}

Figure 7 shows the temperature dependence of the electrical conductivity (Arrhenius plot) for P-51, PG, TBS, and styrene-acryl (St-Ac) resin. In all samples, the conductivity increases almost linearly with temperature, especially in the range above $60^{\circ} \mathrm{C}$, showing semiconductor-like behavior. The conductivity of St-Ac polymer is lower than that of the CCAs by two to three orders of magnitude. The temperature gradient is highest in P-51 and then decreases in sequence of TBS and PG. The increase in conductivity at $\sim 100^{\circ} \mathrm{C}$, relative to that at room temperature, is three orders of magnitude in P-51, compared to two orders of magnitude in TBS and one order of magnitude in PG. As judged from our model, a higher temperature gradient is obviously better for the charge control ability.

The above results clearly indicate that the CCA that exists at the toner/carrier interface (see Figure 1) forms a conductive channel for the charge carrier as soon the toner/ carrier interface is instantaneously heated upon collision above $100^{\circ} \mathrm{C}$. This leads to the efficient charging so that the toner is quickly charged and saturated.

Subsequently additional measurements of temperature dependence of electrical conductivity have been carried out on CCAs other than P-51, PG, and TBS. The following CCAs gave quite similar results to those of P-51, PG, and TBS: azo-Fe complex (T77 from Hodogaya Chemical), ${ }^{19-24}$ azo-Cr complex (S34 from Orient Chemical Industries), ${ }^{25}$ and Al-3,5-tert-butylsalicylate (E88 from Orient Chemical Industries). ${ }^{26}$

\section{Determination of the Charge Carrier}

Figure 8 shows the Seebeck potential as a function of time. P-51 and PG exhibit positive potentials, indicating that the charge carriers are electrons. On the other hand, the thermoelectric potential of TBS was too small to measure in the present experiment.

\section{Work Function of the Fresh Carrier and CCA-Coated Carrier}

The fresh and CCA-covered toners exhibited work functions of 5.05 and $4.90 \mathrm{eV}$, respectively. The difference in work function indicates that the CCA embedded in the toner is partly transferred to the surface of the fresh carrier during the triboelectrification to modify the work function of the fresh carrier.

\section{Wetting Properties of CCA}

Based upon the preceding discussions, CCA works as a conductive mediator upon triboelectrification to facilitate the charge transfer between the toner and the carrier. Another

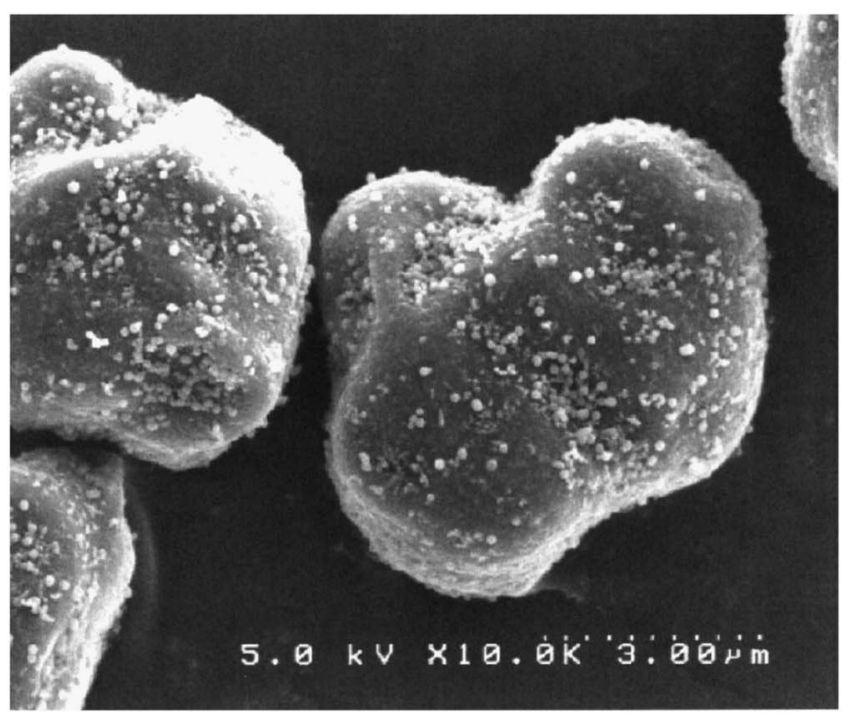

(a)

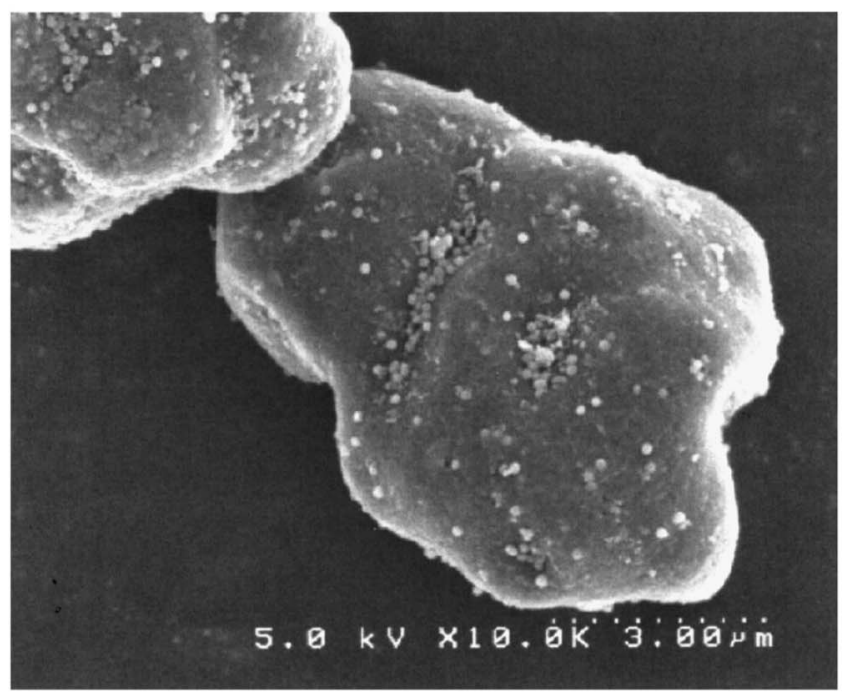

(b)

Figure A1. Scanning electron microscope (SEM) pictures showing the surface appearance of toner particles in a single component system (a) the initial state before use and (b) the state after several ten thousands printing, showing that the silica particles are present only in the shadow.

important function of the CCA is the wetting effect of CCA to minimize the surface tension and also to maximize the contact area of the toner/carrier interface. This further accelerates the charge transfer efficiency. The present function is compared to that of flux used often in solid-state reactions in powders or in metal/metal soldering.

\section{CONCLUSIONS}

We have proposed a novel charge control mechanism of CCA that assumes an appreciable temperature increase at the toner/carrier interface due to triboelectrification. We have rationalized the present model on the basis of the experimental results below.

1. The local temperature at the toner/carrier interface rises above $100^{\circ} \mathrm{C}$ due to triboelectrification. 
2. The CCA sandwiched by toner and carrier upon collision is accordingly heated above $100^{\circ} \mathrm{C}$.

3. The CCAs exhibit a semiconductor-like temperature dependence of electrical conductivity, and the conductivity around $100^{\circ} \mathrm{C}$ is higher by one to three orders of magnitude as compared with that at room temperature. We conclude that the CCA forms a conductive channel as soon as the toner/carrier impact takes place, through which carrier flow occurs effectively to charge the toner, resulting in charge saturation.

\section{ACKNOWLEDGMENTS}

The authors would like to express their sincere thanks to T. Oguchi of Morimura Chemicals for the preparation of toners and also for stimulating discussion. Thanks are also due to S. Imanaga of Canon Inc. for discussion.

\section{APPENDIX: SURFACE APPEARANCE OF TONER PARTICLES}

As supporting material for the temperature increase of toners at the contact between toner and sleeve, some scanning electron microscope pictures of toners in a singlecomponent system are shown in Figures A1a and A1b. Figure Ala shows the initial surface appearance of the fresh toner which is covered with silica particles. On the other hand, the silica particles are embedded in the toners after several ten thousands' printing as shown in Figure A1b, and the silica particles can only be recognized in the shadow of the toner surface. This is presumably due to the local heating of the toner surface caused by triboelectrification with the sleeve. This suggests that the local temperature at the toner/ sleeve contact is also increased above $100^{\circ} \mathrm{C}$.

\section{REFERENCES}

${ }^{1}$ L. B. Schein, "Theory of toner charging", J. Imaging Sci. Technol. 37, 1-4 (1993)

${ }^{2}$ L. B. Schein, "Electric field theory of toner charging", J. Imaging Sci. Technol. 44, 475-483 (2001)

${ }^{3}$ R. J. Nash, M. L. Grande, and R. N. Muller, "CCA Effects on the triboelectric charging properties of a two-component xerographic developer", Proc. IS\&T's NIP 17 (IS\&T, Springfield, VA, 2001) pp. $358-364$.

${ }^{4}$ A. Suka, M. Takeuchi, K. Suganami, and T. Oguchi, "Toner charge generated by CCA particles at the interface between toner and carrier", J. Imag. Soc. Jpn. 45, 127-132 (2006).

${ }^{5}$ J. Guay, J. E. Ayala, and A. F. Diaz, "The question of solid-state electron transfer in contact charging between metal and organic materials", Chem. Mater. 3, 1068-1073 (1991).

${ }^{6}$ J. Mizuguchi, A. Hitachi, Y. Sato, and K. Uta, "A study on the charge control mechanism", Proceedings of the ICJ 2006 Fall Meeting (The
Imaging Society of Japan, Tokyo, 2006) pp. 121-124.

${ }^{7}$ J. Mizuguchi, A. Hitachi, Y. Sato, and K. Uta, "A study on the charge control mechanism”, Proc. IS\&T's NIP23 (IS\&T, Springfield, VA, 2007) pp. 121-124.

${ }^{8}$ J. Mizuguchi, Y. Sato, K. Uta and K. Sato, "Benzyltributylammonium 4-hydroxynaphthalene-1-sulfonate", Acta Crystallogr. E63, o2509-o2510 (2007).

${ }^{9}$ K. Uta, Y. Sato, and J. Mizuguchi, "Crystal structure of a charge control agent of the quarternary ammonium salt and its electrical properties", Proc. ICJ 2007 Spring Meeting (The Imaging Society of Japan, Tokyo, 2007) pp. 83-86.

${ }^{10}$ S. Iwata, A. Hitachi, T. Makino, and J. Mizuguchi, " $n$-Propyl gallatechloroform (3/0.5)", Acta Crystallogr. E61, o2587-o2589 (2005).

${ }^{11}$ J. Mizuguchi, A. Hitachi, S. Iwata, and T. Makino, " $n$-Propyl gallateacetonitrile (2/1)", Acta Crystallogr. E61, o2593-02595 (2005).

${ }^{12}$ A. Hitachi, T. Makino, S. Iwata, and J. Mizuguchi, " $n$-Propyl gallatedichloromethane (3/0.5)”, Acta Crystallogr. E61, o2590-o2592 (2005).

${ }^{13} \mathrm{~J}$. Mizuguchi, "3,5-Di-tert-butyl-2-hydroxybenzoic acid", Acta Crystallogr. E59, o1530-o1531 (2003).

${ }^{14}$ J. Mizuguchi, K. Hino, and K. Tojo, "Strikingly different electronic spectra of structurally similar perylene imide compounds", Dyes Pigm. 70, 126-135 (2006).

${ }^{15} \mathrm{~J}$. Mizuguchi and K. Tojo, "Crystal structure of $N, N^{\prime}$-bis(2-(4pyridyl)ethyl)-perylene-3,4:9,10-bis(dicarboximide)", Z. Kristallogr. New Cryst. Struct. 217, 247-248 (2002).

${ }^{16} \mathrm{~J}$. Mizuguchi and K. Hino, " $N, N$ '-Bis[2-(4-pyridyl)ethyl]perylene3,4:9,10-bis (dicarboximide)phenol disolvate", Acta Crystallogr. E61, o669-o671 (2005).

${ }^{17} \mathrm{~K}$. Hino and J. Mizuguchi, " $N, N^{\prime}$-Bis[2-(4-pyridyl)ethyl]perylene3,4:9,10-bis(dicarboximide) $m$-cresol disolvate", Dyes Pigm. E61, 0672-0674 (2005).

${ }^{18}$ K. Seeger, Semiconductor Physics, 3rd ed. (Springer Verlag, Berlin, 1985).

${ }^{19}$ Y. Sato, K. Uta, and J. Mizuguchi, "Crystal structure of an azo Fecomplex used as a charge control agent and its electrical properties", Proc. ICJ 2007 (The Imaging Society of Japan, Tokyo, 2007) pp. 87-90.

${ }^{20}$ J. Mizuguchi, Y. Sato, and K. Uta, "Ammonium bis\{ 3-anilinocarbonyl1-[(5-chloro-2-oxidophenyl)diazenyl] -2-naphtholato\} ferrate(III) methanol solvate", Acta Crystallogr. E63, m1327-m1328 (2007).

${ }^{21} \mathrm{~J}$. Mizuguchi, K. Uta, and Y. Sato, "Ammonium bis\{3-anilinocarbonyl-1[(5-chloro-2-oxidophenyl)diazenyl] -2-naphtholato\} ferrate(III) acetone solvate", Acta Crystallogr. E63, m1329-m1330 (2007).

${ }^{22}$ Y. Sato, K. Uta, and J. Mizuguchi, "Crystal structure of an azo Fecomplex sodium salt used as a charge control agent and its electrical properties", Proc. ICJ 2007 Fall Meeting (The Imaging Society of Japan, Tokyo, 2007) pp. 113-116.

${ }^{23}$ J. Mizuguchi, Y. Sato, and K. Uta, "Poly[bis(acetone)bis $[\mu-4-[(5-$ chloro-2-oxidophenyl)diazenyl]-3-oxido- $N$-phenylnaphthalene-2-carboxamidato]sodium(I)ferrate(III)]", Acta Crystallogr. E63, m1377-m1378 (2007).

${ }^{24}$ Y. Sato, K. Uta, and J. Mizuguchi, "Diacetonitriletetrakis $\{\mu 2-3-$ anilinocarbonyl-1-[(5-chloro-2-oxidophenyl)diazenyl]-2-naphtholato\} tetraaquadiiron(III)disodium(I) dehydrate", Acta Crystallogr. E64, m240-m241 (2008).

${ }^{25} \mathrm{~S}$. Ito, Y. Sato, and J. Mizuguchi, "Poly[bis(acetone- $\left.\kappa \mathrm{O}\right) \operatorname{bis}\left\{\mu_{3}-1-\right.$ [(5-chloro-2-oxidophenyl) diazenyl]-2-naphtholato- $\left.\kappa^{4} O: O, O^{\prime}: O^{\prime}\right\}$ sodium(I)chromium(III)]”, Acta Crystallogr. E64, m333-m334 (2008).

${ }^{26}$ Y. Kamei and J. Mizuguchi, "Crystal structure of a salicylic acid derivative Al-complex used as a charge control agent and its electrical properties", Proc. Pan-Pacific Imaging Conference 2008 (The Imaging Society of Japan, Tokyo, 2008) 
(C)2008 Society for Imaging Science and Technology (IS\&T)

All rights reserved. This paper, or parts thereof, may not be reproduced in any form without the written permission of IS\&T, the sole copyright owner of

The Journal of Imaging Science and Technology.

For information on reprints or reproduction contact

Donna Smith

Production Editor

The Journal of Imaging Science and Technology

Society for Imaging Science and Technology

7003 Kilworth Lane

Springfield, Virginia 22151 USA

703/642-9090 extension 107

703/642-9094 (fax)

dsmith@imaging.org

www.imaging.org 